\title{
EFFECTS OF SEXTUPOLE TIME DEPENDENCE ON THE LHC DYNAMIC APERTURE
}

\author{
Y. Papaphilippou, F. Schmidt and F. Zimmermann, CERN, Geneva, Switzerland
}

\section{Abstract}

A primary concern regarding the LHC dynamic aperture is the time dependence of persistent-current sextupole fields in the superconducting magnets. Decaying slowly during injection, these fields are reinduced rapidly at the start of the acceleration ("snap-back"). If uncompensated, this snap-back would cause a chromaticity change by some 130 units. We investigate how this time dependence and the ramp rate affect the stability of particle motion and we evaluate the efficiency of different correction schemes.

\section{INTRODUCTION}

Superconducting magnets provide high magnetic fields at low operating costs. Therefore, they are the magnets of choice for the present and next-generation highest-energy proton storage rings, e.g., for the Large Hadron Collider (LHC) now under construction at CERN [1]. The superconducting magnets exhibit large nonlinear field errors. These are partly caused by the geometry of the superconducting coils, and partly due to "persistent currents" (p.c.), which are eddy currents in the superconducting cable. The nonlinear field errors are expected to limit the LHC "dynamic aperture" (the stable region of phase space where particles are not lost) at injection energy.

In order to ensure an adequate dynamic aperture of $6 \sigma$ as required by the collimation system [1], the effect of the strongest magnet nonlinearities, such as the normal sextupole component, will be compensated by dedicated multipole correctors. At the injection plateau, where the magnet field quality is most critical, the p.c. errors decay in time $[2,3]$. This decay is caused both by a slow flux creep in the superconductor and, more importantly, by a current redistribution between the strands of the superconducting cable. At the start of the acceleration the p.c. fields are reinduced rapidly, a phenomenon called the "snap-back" [2].

During acceleration a new kind of eddy current is induced in the loops formed by the twisted strands in the superconducting cable $[4,5]$. The resulting dynamic field imperfections are proportional to the ramp rate and inversely proportional to the inter-strand resistance.

In this report we employ particle tracking to investigate the influence of the persistent-current decay, the snap-back and the ramp-induced field imperfections on the dynamic aperture, and we estimate the implied tolerances on chromatic correction and ramping speed.

\section{PARAMETERS}

The LHC, a double storage-ring with a circumference of $26.7 \mathrm{~km}$, is designed to collide two $7-\mathrm{TeV}$ proton beams. Relevant parameters at injection energy are compiled in
Table 1. It will take about 7 minutes to inject both LHC beams; a three times longer time period is required for acceleration to top energy.

The tracking simulation with the SIXTRACK code [6] models a time-span of $10^{5}$ turns, which corresponds to approximately $1 \%$ of the total injection period. Initial particle coordinates are chosen equally spaced in the transverse linear Courant-Snyder invariants $I_{x 0}$ and $I_{y 0}$, at a constant ratio of $I_{x 0} / I_{y 0}$ (usually 1 ). The initial transverse momenta are set to zero and the initial energy error is $\delta=1.6 \sigma$ (or $7.5 \times 10^{-4}$ ), roughly $75 \%$ of the rf bucket half size. Twin particles with slightly different initial conditions are tracked in order to determine the onset of chaotic motion by computing the Lyapunov exponent [7]. The tracking is repeated for 10 random seeds of the multipole errors, and we infer both the average and the minimum dynamic aperture over all error seeds. Throughout this report, the dynamic aperture $a$ refers to a simultaneous amplitude in both transverse planes, in units of the rms beam size. The aperture is calculated from the transverse phase space areas $A_{x, y}$ inscribed by a particle during its betatron motion, via $a=\sqrt{\left(A_{x}+A_{y}\right) /(2 \pi)}$.

Our simulation study assumes a realistic set of nonlinear field components for the main dipoles known by the acronym "9712". The strengths of the sextupole field errors for this error set are given in Table 2. Higher multipoles and their variation up to order 11 (not listed in the table) are also taken into account. The simulation is performed for LHC optics version 6, with a 4-integer tune split between the horizontal and vertical plane. The linear optics is considered to be constant. Thus, changes of dipole and quadrupole fields or feeddown from higher-order multipoles for off-center orbit are disregarded.

Table 1: LHC injection parameters.

\begin{tabular}{lcc}
\hline parameter & symbol & value \\
\hline proton energy & $E$ & $450 \mathrm{GeV}$ \\
transv. norm. emittance & $\gamma \epsilon_{x, y}$ & $3.75 \mu \mathrm{m}$ \\
transv. rms beam size in arc & $\sigma_{x, y}$ & $\sim 1.2 \mathrm{~mm}$ \\
rms energy spread & $\sigma_{\delta}$ & $4.7 \times 10^{-4}$ \\
rms bunch length & $\sigma_{z}$ & $13 \mathrm{~cm}$ \\
betatron tunes & $Q_{x, y}$ & $63.28,59.31$ \\
\hline
\end{tabular}

\section{CHROMATICITY}

The decrease of the average sextupole fields due to persistent-current decay at injection energy, if uncompensated, results in a huge chromaticity variation of $\Delta Q_{x}^{\prime} \approx$ +144 , and $\Delta Q_{y}^{\prime} \approx-129$ (where $Q^{\prime}$ is defined as 
$\Delta Q /(\Delta p / p))$. The opposite chromaticity change occurs, in less than 1 minute, during the snap-back. To permanently maintain a small net chromaticity, sextupole correction coils must be powered synchronized with the accelerating cycle. It is of interest to estimate the correction accuracy required.

Table 2: Normal and skew sextupole components in the main LHC dipoles, assumed in this study. The errors are quoted in units of $10^{-4}$, normalized to the main dipole field, for a reference radius of $17 \mathrm{~mm}$. The different rows show the field errors due to p.c. decay, geometry \& iron, persistent currents, and acceleration at the nominal ramp rate of $8 \mathrm{~T} / 20 \mathrm{~min}$, respectively. In each case the first number is the mean change, the second the uncertainty (the rms difference between octants), and the third the rms variation between magnets. The table does not show the multipole errors of order $n=4$ to 11 , which were also included in the simulation.

\begin{tabular}{|c|c|c|c|}
\hline contribution & mean & uncert. & $\mathrm{rms}$ \\
\hline \multicolumn{4}{|c|}{ normal sextupole $b_{3}$} \\
\hline p.c. & -10.7 & 1.1 & 0.3 \\
\hline p.c. decay & 3.2 & 0.4 & 1.0 \\
\hline geometry\& iron & 2.9 & 0.9 & 1.4 \\
\hline ramp & 1.0 & 0.2 & 0.3 \\
\hline \multicolumn{4}{|c|}{ skew sextupole $a_{3}$} \\
\hline p.c. & 0.0 & 0.0 & 0.2 \\
\hline p.c. decay & 0.0 & 0.3 & 0.7 \\
\hline geometry\& iron & 0.0 & 0.9 & 0.4 \\
\hline ramp & 0.0 & 0.2 & 0.3 \\
\hline
\end{tabular}

Figure 1 presents the simulated dynamic aperture at the start of LHC injection as a function of equal and oppositesign chromaticity in the two transverse planes. The case of opposite-sign chromaticity, which naturally occurs during p.c. decay or snap-back, shows a stronger effect on the dynamic aperture. In either case a chromaticity of a few units seems acceptable. Larger chromatic variations could seriously reduce the dynamic aperture which scales roughly inversely with the chromaticity.

In Fig. 2 we depict the dynamic aperture after the persistent current decay, near the end of the injection plateau. Comparing with Fig. 1 (top) shows that the effect of the p.c. decay on the dynamic aperture is insignificant, provided that the chromaticity is held constant. The smallness of this effect can be attributed to the fact that, firstly, an increase of either random or systematic sextupole components leads to a comparable reduction of the dynamic aperture (see Fig. 3) and that, secondly, during the p.c. decay the systematic sextupole decreases while the random component increases, by roughly $40 \%$ and $22 \%$, respectively (see Table 2).

A frequency map analysis $[8,9]$ of the two cases, before and after p.c. decay, reveals that resonances are encountered at approximately the same amplitudes as illustrated in Fig. 4. This implies that these resonances remain fixed
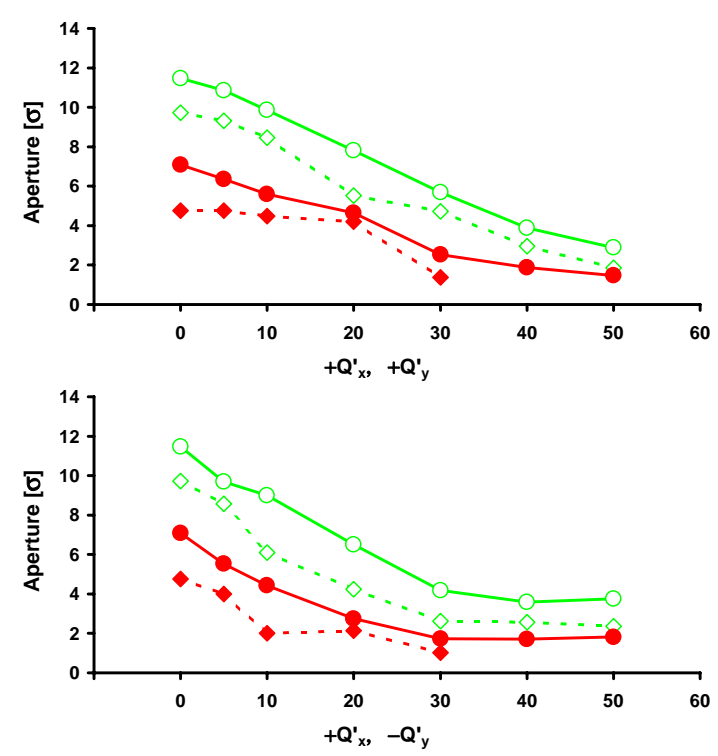

Figure 1: Dynamic aperture as a function of uncorrected chromaticity, at the start of the injection plateau. Top: equal sign for horizontal and vertical chromaticity, bottom: opposite sign. Shown are the mean (circles) and the minimum (diamonds) amplitudes beyond which particles are lost in less than $10^{5}$ turns (open symbols) and beyond which the particle motion is found to be chaotic (closed symbols). The chromaticity was varied by changing the strength of the main sextupoles.

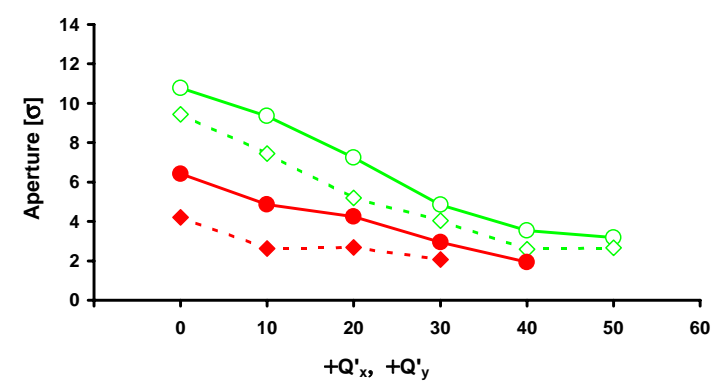

Figure 2: Dynamic aperture as a function of uncorrected chromaticity, at the end of the injection plateau (after the p.c. decay), considering equal horizontal and vertical chromaticity. The plotting symbols are the same as in Figure 1.

during the entire decay process. Hence, the dynamic aperture should not be influenced by the time dependence.

A correction of the chromaticity change due to p.c. decay (or snap-back) can be performed either locally with the spool pieces in the dipole magnets or with the main lattice sextupoles. Figure 5 shows that the two correction methods would provide about the same aperture.

It should be mentioned that a large negative chromaticity may induce the fundamental-mode head-tail instability. To insure beam stability at nominal current, the chromaticity should be larger than -1 unit. On the other hand for positive values, the chromaticity is limited to a few units to avoid the $m=1$ head-tail mode [10] and to restrict the tune footprint. 


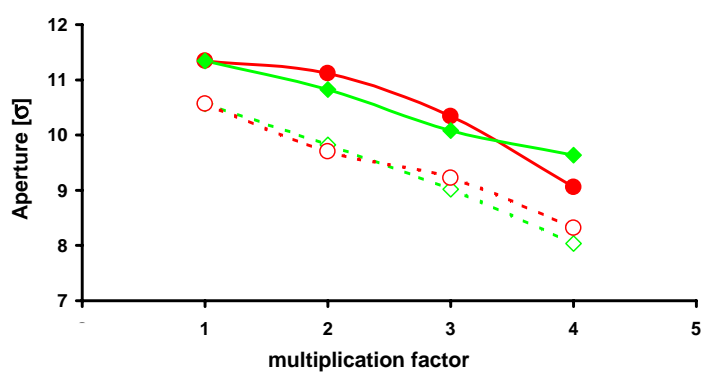

Figure 3: Dynamic aperture as a function of random (diamonds) and systematic (circles) sextupole components in units of the nominal strength. Solid lines represent the mean value, dashed lines the minimum over all seeds.

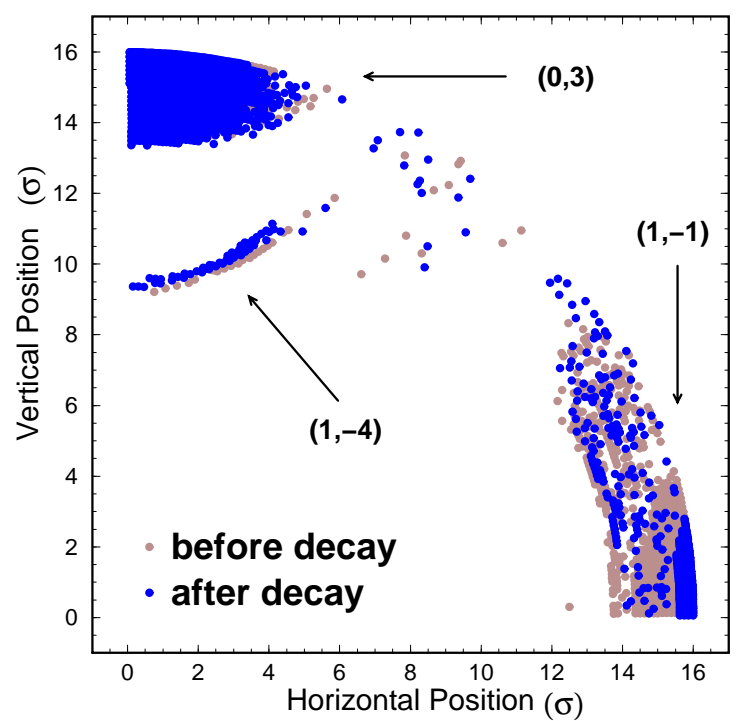

Figure 4: Initial amplitudes of particles locked to three strong resonances, determined by tracking and frequency map analysis [8]

\section{RAMP RATE}

The additional imperfections caused by interstrand coupling during acceleration are proportional to the ramp rate (see Table 2 for the change in sextupole field). Figure 6

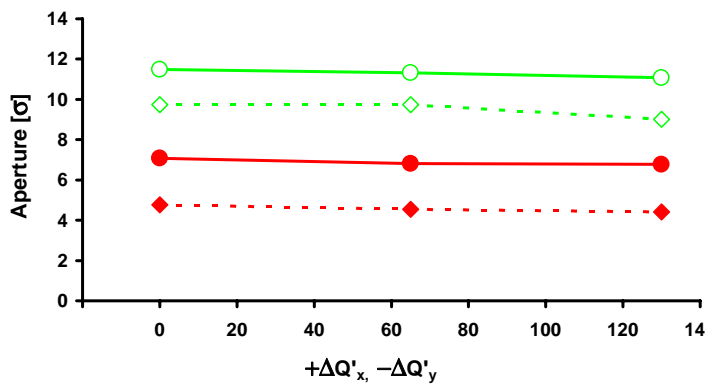

Figure 5: Dynamic aperture after half and full snapback corresponding to $\Delta Q_{y}^{\prime} \approx-\Delta Q_{x}^{\prime} \approx+65$ and +130 units, respectively and its correction with the lattice sextupoles (instead of using the spool pieces). The plotting symbols are the same as in Figure 1 demonstrates that the dynamic aperture is not much affected by these additional field errors, up to ramp rates four times larger than nominal.

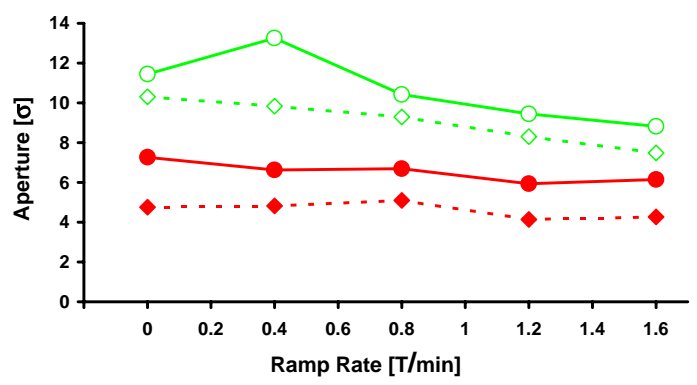

Figure 6: Dynamic aperture during acceleration as a function of ramp rate, close to the injection energy. The plotting symbols are the same as in Figure 1. The chromaticity is corrected for all points.

\section{CONCLUSIONS}

Assuming the linear optics stays constant, we found that the time variation of the higher-order field errors-induced by p.c. decay at injection or by the subsequent snap-backhas only minor effects on the LHC dynamic aperture, as long as the overall chromaticity is corrected and remains within a few units. Under the same assumptions, the dynamic aperture during acceleration shows little dependence on the ramp rate. Correcting the chromaticity with the main arc sextupole magnets is about as effective as correcting it with the sextupole spool pieces in the bending magnets.

\section{ACKNOWLEDGEMENTS}

We thank L. Bottura and R. Wolf from the CERN LHC division for discussions on the persistent-current decay and the snap-back, and J. P. Koutchouk for helpful suggestions.

\section{REFERENCES}

[1] The LHC Study Group, CERN/AC/95-05 (1995).

[2] L. Bottura, et al. , 1996 Applied Supercon. Conference.

[3] K.H. Meß, P. Schmüser, and S. Wolf, "Superconducting Accelerator Magnets”, World Scientific (1996).

[4] A.P. Verwej and R. Wolf, CERN AT-MA/AV 94-07 (1994).

[5] A. Faus-Golfe, LHC Project Note 9 (1995).

[6] F. Schmidt, CERN-SL-94-56-AP (1994).

[7] F. Schmidt, et al. , Part. Accel. 35, 249 (1991).

[8] J. Laskar, Physica D 67, 257 (1993); J. Laskar and D. Robin, Part. Acc. 54, 183 (1996).

[9] Y. Papaphilippou, this conference.

[10] S. Berg, LHC Project Report 100 (1997). 